\title{
Dynamic Models of Dry Electrostatic Precipitator in a 1000MW Coal-fired Plant
}

\author{
Zhongwei Wang ${ }^{1}$, Zhi-gang $\mathrm{Su}^{1 *}$, Xiaojin $\mathrm{Zhu}^{2}$, Yongsheng Hao ${ }^{1}$ \\ ${ }^{1}$ School of Energy and Environment, Southeast University, Nanjing, Jiangsu Province, 210096, China \\ ${ }^{2}$ Zhejiang Zheneng Taizhou Second Electric Power Generation Co. Ltd., Taizhou, Zhejiang Province, 318000, China
}

\begin{abstract}
Dynamic model is the foundation to achieve feedback control of Electrostatic Precipitator (ESP) so as to reduce dust emission concentration and high power consumption. This paper investigates the dynamic modeling of outlet dust concentration of a dry electric precipitator with five electric field structure in a $1000 \mathrm{MW}$ power plant. The secondary current of the high-frequency power supply of the last two electric fields of the ESP are taken as control inputs, whereas the outlet dust concentration is taken as output. With increasingly stepping the secondary current at four typical load points, experimental data of outlet dust concentration are collected to identify parameters of dynamic models after fixing its structure based on an immune genetic algorithm. The experimental results suggest that the established dynamic models can capture the real dynamics of ESP and have high accuracy.
\end{abstract}

\section{Introduction}

The annual dust emission from coal-fired power plants is the main source of dust pollutants in atmospheric environment in China. In order to effectively curb pollutant emissions from thermal power plants and improve air quality, the Ministry of Environmental Protection has repeatedly raised environmental standards. Electrostatic precipitator has occupied an absolute position in the field of thermal power dust control for a long time. With the continuous improvement of environmental protection standards, thermal power plants have to increase the output of electrostatic precipitators in order to ensure the dust removal effect during operation, which will inevitably increase the power consumption of electric precipitators. Under normal circumstances, the electricity consumption of the electrostatic precipitator accounts for about 3\% $5 \%$ [1] of the electricity used by the power plant. The reduction of electrostatic precipitator power consumption has caused the thermal power plant to attach great importance.

In order to improve the dust removal effect and achieve energy-saving operation, the domestic electrostatic precipitator power supply is generally replaced with a high-frequency power supply. In addition, research institutes and power plants have done a lot of research work, such as optimization control. Although the idea of energy-saving optimization and control of the outlet concentration of ESP has been proposed [2], the closed loop control of concentration feedback has not been put into used. The dust removal efficiency of electrostatic precipitator is affected by many factors, such as smoke properties, smoke volume, supply voltage and current, negative pole and positive rapping effect, airflow distribution, etc. [3]. The mathematical model of electrostatic precipitator is difficult to be accurately established by mechanism modelling. There is no dust concentration measurement point at the entrance of the electrostatic precipitator and between the electric fields, so advanced control methods are difficult to apply.

At present, the energy-saving optimization of electrostatic precipitators in coal-fired plant generally uses an open-loop control method, that is, the operating parameter setting values of the high-frequency power supply are adjusted according to the unit load. Some scholars have proposed energy-saving optimization control of electrostatic precipitator based on intelligent algorithm [3-6]. Applying the neural network to establish the outlet concentration-supply voltage model of the electrostatic precipitator, the basic genetic algorithm is used to optimize the energy-saving parameters of the high-voltage power supply. However, the established concentration-voltage model is essentially a static model, which cannot meet the operation requirements of the variable load variable operating conditions of the coalfired plant.

In this paper, in order to realize the concentration closed-loop control of electrostatic precipitator, the transfer function models of the secondary current of the last two electric fields high-frequency power supply to the electrostatic precipitator outlet concentration at four typical load points were established by means of experimental modeling. The load point range can cover the load fluctuation range of the unit during normal operation. Established models are suitable for the unit variable working condition. The transfer function models

$\overline{{ }^{*} \text { Corresponding author's e-mail: zhigangsu@seu.edu.cn }}$ 
are verified by the same current control. The results show that the established models are reliable.

\section{Working principle of electrostatic precipitator}

The electrostatic precipitator is a dust removing device that uses a strong electric field generated by a highvoltage power source to ionize the gas, thereby charging the suspended dust particles, and separating the suspended dust particles from the gas under the action of the electric field force. The dust removal principle of the electrostatic precipitator is shown in Figure 1.

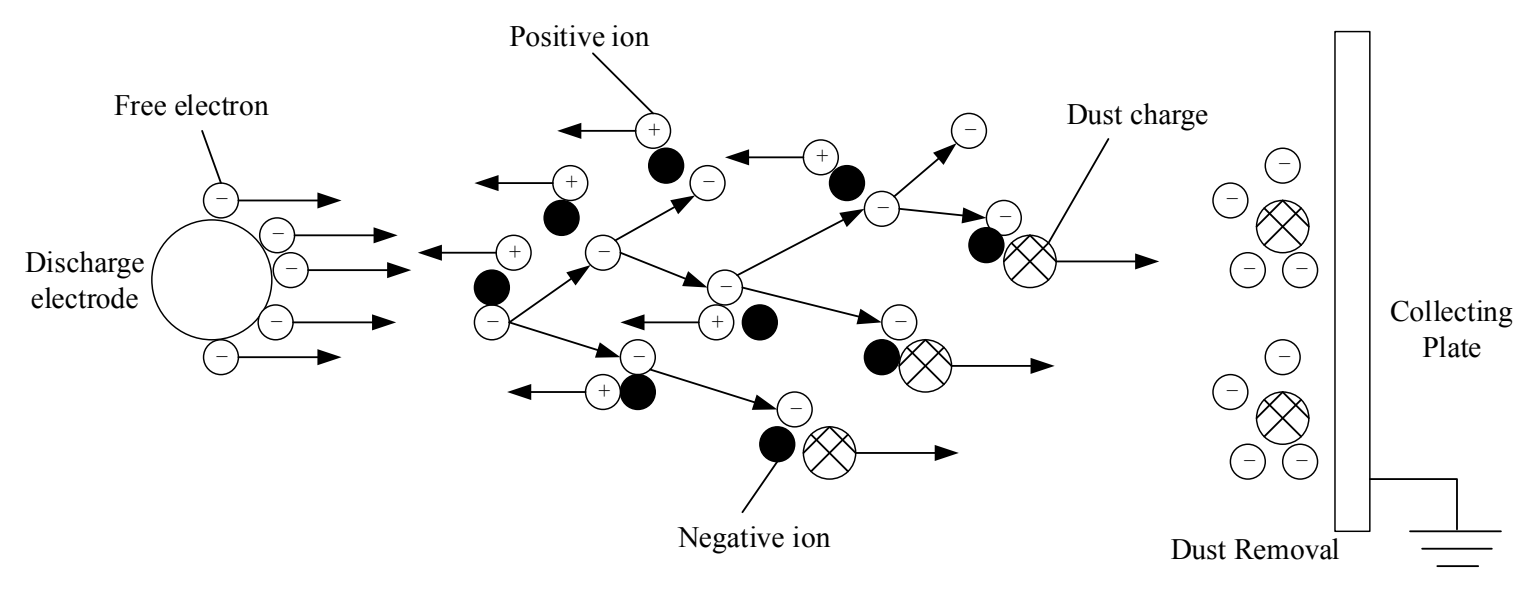

Figure 1. Schematic diagram of electrostatic precipitator

The dust removal process of the electrostatic precipitator can be divided into four processes [7] :

(1) Ionization of gas. The high-voltage direct current applied by the corona pole generates a strong electric field in the corona region, causing corona discharge of the dust-containing gas entering the electric field, and the gas is ionized to generate positive ions and free electrons. Under the action of the electric field force, the free electrons collide with the gas atoms, and a free electron is added.

(2) Charge of dust. A large amount of free electrons, positive ions and negative ions generated after gas ionization move under the action of the electric field Coulomb force and collide with the dust, and adhere to the dust particles to charge the dust.

(3) Collection of charged dust. A large amount of negatively charged dust moves to the dust collecting pole under the action of the electric field Coulomb force, reaches the dust collecting pole and releases the charged charge and deposits on the dust collecting pole. A small amount of positively charged dust in the corona area is deposited on the discharge electrode.

(4) Rapping and cleaning. After the dust on the surface of the electrode reaches a certain thickness, the dust on the electrode is collected by mechanical vibration and discharged from the lower ash hopper.

\section{Transfer function identification method based on immune genetic algorithm}

Immune genetic algorithm [8] is an improved genetic algorithm based on biological immune mechanism, which is a genetic algorithm with immune function. Compared with genetic algorithm, immune genetic algorithm has faster search speed and higher overall search ability. The flow chart of immune genetic algorithm is shown in Figure 2.

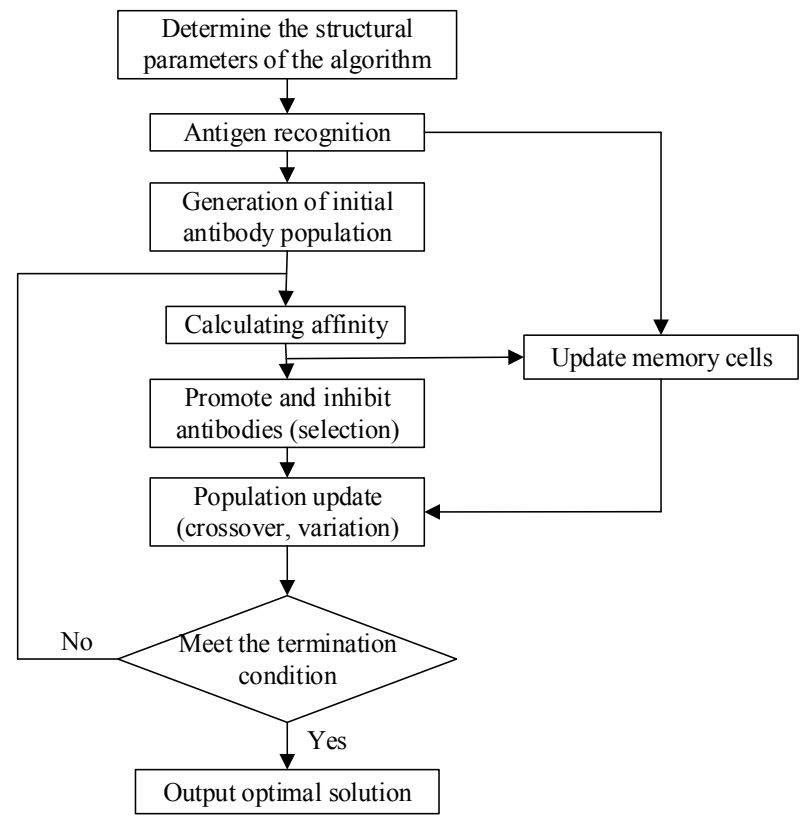

Figure.2 Flow chart of immune genetic algorithm

In this paper, the average value of the secondary current of all the electric chambers of last two electric fields is taken as input, and the dust concentration of the electrostatic precipitator outlet is taken as the output. The purpose of identification is to establish the transfer function model of the secondary electric current of the 
last two electric fields of the electrostatic precipitator to the outlet dust concentration. The specific implementation steps are:

(1) Determine the structural parameters of the algorithm. For example, evolutionary algebra $\mathrm{G}=500$, antibody population number $N=100$, memory cell number $N m=5$, crossover frequency $P_{c}=0.8$, mutation frequency $P m=0.01$, and the like.

(2) Antigen recognition. Select the objective function of the problem to be solved as the antigen. The transfer function model structure to be identified is taken:

$$
G_{d 4,5}(s)=\frac{C_{d}(s)}{I_{4,5}(s)}=\frac{K}{1+T s} \mathrm{e}^{-\tau s},
$$

where, $C_{d 4,5}(s)$ is the outlet dust concentration $\left(\mathrm{mg} / \mathrm{Nm}^{3)}\right.$ of ESP, $I_{4,5}(s)$ is the average value $(\mathrm{mA})$ of the secondary current of all the electric chambers of the electric field of last two electric fields. Therefore, the optimization variables are determined to be $K, T$ and $\tau$.

(3) Initial antibody population generation. The combination of parameters to be optimized is used as an antibody, and a certain number of combinations are randomly generated according to the actual interval of the solution to form an initial antibody population and initial memory cells, which are updated with each other. The value range of the model parameters in this paper is set to: $\tau \in[15,40], \quad K \in[-0.5,0.5]$ and $T \in[0,100]$.

(4) Calculate the affinity. The affinity of the antigen to the antibody can be equated to the fitness function. Define the fitness function:

$$
f=\sum_{i=1}^{N}\left|\frac{\Delta C_{d}}{C_{d}}\right|,
$$

where, $C_{d}$ is the actual smoke concentration, $\Delta C_{d}$ is the difference between the actual value and the calculated value of the model.
(5) Update memory cells. The initial population is arranged in descending order according to $\left(A_{g}\right)_{i}$, and antibodies with high antigen affinity are added to the memory cells.

(6) Promotion and inhibition of antibodies. Antibody concentration $C_{i}$ is calculated and antibody selection is performed in conjunction with $\left(A_{g}\right)_{i}$. The criteria for selecting antibodies consist of two parts: antibody affinity and concentration inhibitor:

$$
E_{i}=\lambda\left(A_{g}\right)_{i}+(1-\lambda) \mathrm{e}^{-\mu C_{i}},
$$

where, $C_{i}$ is the antibody concentration, $\lambda$ and $\mu$ is the weighting factor, taking $\lambda=0.7, \mu=1.25$.

The antibody concentration $C_{i}$ is defined as:

$C_{i}=\frac{\text { the number of the affinity with antibody } i \text { is greater than } \theta}{N}$,

where, $\theta$ is the affinity constant, taking $\theta=0.9$.

(7) Generation of new antibodies. A new population is obtained by performing crossover and mutation operations on selected antibodies in the antibody population.

(8) Determine the termination condition. In this paper, the largest evolutionary algebra is used as the termination condition, and the last optimal antibody is output.

\section{Testing and modelling}

Taking the electrostatic precipitator of $1000 \mathrm{MW}$ Coalfired power plant as the research object, the electrostatic precipitator has multiple electric field structure, equipped with six channels, five electric fields and 30 highfrequency power supplies. The plane diagram is shown below.

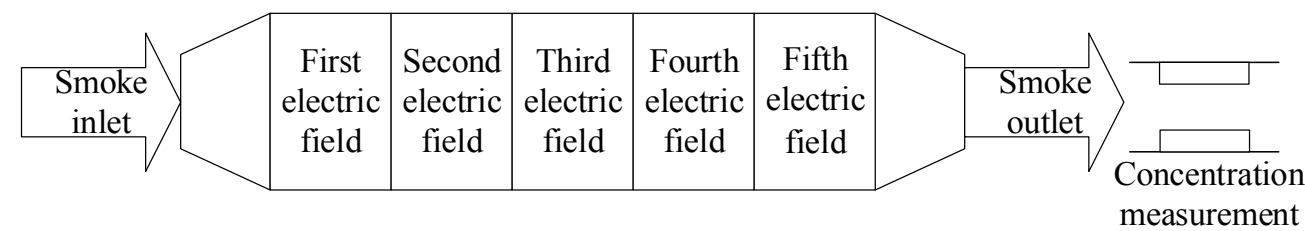

Figure.3 Schematic plan view of ESP with five electric field structure

In order to study the dynamic characteristics of dry electrostatic precipitator, dynamic characteristics tests were carried out on the controlled objects of outlet dust concentration at the ESP. Specifically tested at four load points of $650 \mathrm{MW}, 750 \mathrm{MW}, 850 \mathrm{MW}$ and $980 \mathrm{MW}$. At each load point, the static characteristic test of the first three electric fields was firstly carried out, adjusting the operating parameters of the high-frequency power supply of the first three electric fields to made the power supply energy-saving operation on the basis of ensuring that the outlet concentration does not exceed the standard. Then, the step disturbance test of the secondary current of the last two electric fields was carried out. According to the obtained experimental data, the transfer function models of the secondary current of the last two electric fields to the outlet dust concentration were fitted by immune genetic algorithm, and the root mean square error was used to evaluate the accuracy of the identified transfer function. The first three electric fields operating parameters and model identification results of each load point are shown in Table 1. 
Table 1. The first three electric fields operating parameters and model identification results

\begin{tabular}{cccccc}
\hline Load (MW) & $\begin{array}{c}\text { First electric field } \\
\text { current }(\mathrm{mA})\end{array}$ & $\begin{array}{c}\text { Second electric field } \\
\text { current }(\mathrm{mA})\end{array}$ & $\begin{array}{c}\text { Third electric field } \\
\text { current }(\mathrm{mA})\end{array}$ & $\begin{array}{c}\text { Last two electric field } \\
\text { transfer function } \\
\text { model }\end{array}$ & $\begin{array}{c}\text { Root mean } \\
\text { square error }\end{array}$ \\
\hline 650 & 229.3271 & 237.4278 & 191.1384 & $\frac{-0.015}{1+62.51 s} \mathrm{e}^{-24 s}$ & 0.5153 \\
750 & 281.3721 & 288.1323 & 237.3769 & $\frac{-0.0143}{1+65.29 s} \mathrm{e}^{-30 s}$ & 0.3553 \\
850 & 368.1132 & 377.1015 & 377.1704 & $\frac{-0.013}{1+30.3 s} \mathrm{e}^{-21 s}$ & 0.693 \\
980 & 468.5187 & 428.0315 & 427.2872 & $\frac{-0.01545}{1+62.05 s} \mathrm{e}^{-27 s}$ & 0.3776 \\
\hline
\end{tabular}

In order to verify the accuracy of the process model, the model output of each load point is compared with the measured dust concentration under the same control.

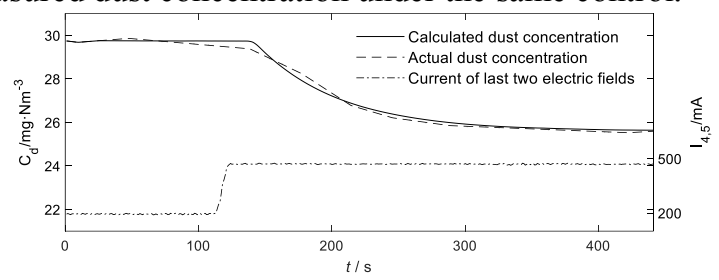

Figure.4 Model output and measured dust concentration under 650MW

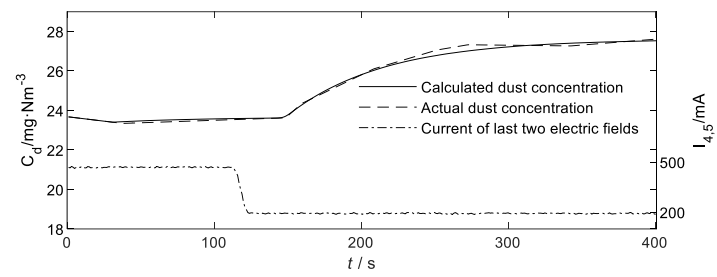

Figure.5 Model output and measured dust concentration under $750 \mathrm{MW}$

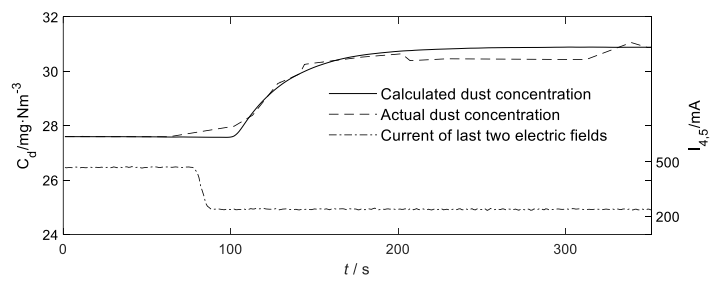

Figure.6 Model output and measured dust concentration under $850 \mathrm{MW}$

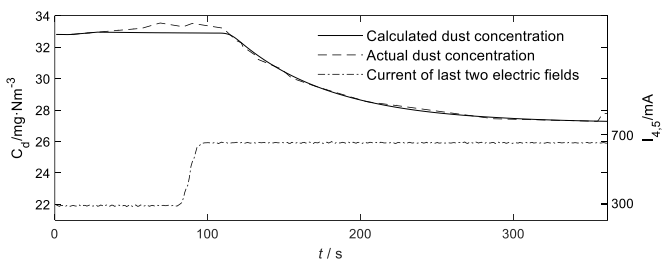

Figure.7 Model output and measured dust concentration under 850MW

It can be seen from the figure 4-7 that the established models have higher accuracy under the load of 650MW, $750 \mathrm{MW}$ and $980 \mathrm{MW}$. Under the $850 \mathrm{MW}$, before the secondary current step of the last two electric fields decreases, the dust concentration of the ESP outlet is increased by other disturbances, and the response is also affected by other disturbances, so the accuracy of the identified model under $850 \mathrm{MW}$ is not very high.

\section{Conclusion}

In this paper, for the dry electrostatic precipitator with five electric field structure, immune genetic algorithm is used to establish the transfer function models of the secondary current of high-frequency power supply of the last two electric fields to the outlet dust concentration by experimental modeling. The established models output is close to the actual value and the established models have high precision. Results of model identification show that electrostatic precipitator dust removal process has dynamic characteristics of large time lag, large inertia and many interferences. The established dynamic models have important theoretical value for the closed-loop control of ESP outlet dust concentration.

\section{References}

1. Li, J. (2014) Design and development of electric dust removal energy-saving optimization control system. China High-tech Enterprise, 9: 71-73.

2. Durga Prasad, N. V. P. R., Lakshminarayana, T., Narasimham, J. R. K., Verman, T. M., Krishnam Raju, C. S. R. (1999) Automatic control and management of electrostatic precipitator. IEEE Transactions on Industry Applications, 35(3): 0-567.

3. Li, D., Tian, L., Liu, S. (2006) Optimized control of the working voltage for electrostatic precipitator based on intellective method. Energy Saving Technology, 24(6): 538-541.

4. Li, Z., Yan, M. (2012) Optimized control system for electrostatic precipitator high voltage power supply. Electric Drive, 42(2): 65-68.

5. Li, Z., Shao, C., An, Y., et al. (2013) Energy-saving optimal control for a factual electrostatic precipitator with multiple electric-field stages based on GA. Journal of Process Control, 23(8):1041-1051.

6. Li, Z. (2014) High Voltage Power Supply Design and Energy-Saving Optimal Control for High 
Efficiency Electrostatic Precipitator. Doctoral dissertation, Dalian: Dalian University of Technology. pp. 99-119.

7. Chen, N. (2015) The Research of Control System on High Voltage Electrostatic Precipitation. Doctoral dissertation, Xi'an: Xi'an Technological University. pp. 10-12.

8. Gao, F., Wang, W., Yang, X. (2016) Parameters turning and optimization for variable-gain PI controller of wind turbine based on immune genetic. Journal of Power Engineering,36(1): 22-29. 